九州大学学術情報リポジトリ

Kyushu University Institutional Repository

\title{
Computer Analysis of Lysozyme-catalyzed Reaction with Chitooligosaccharide
}

\section{Masaki, Atsushi}

Laboratory of Sericultural Chemistry, Faculty of Agriculture, Kyushu University

Aso, Yoichi

Laboratory of Sericultural Chemistry, Faculty of Agriculture, Kyushu University

Koga, Daizo

Laboratory of Sericultural Chemistry, Faculty of Agriculture, Kyushu University

Hayashi, Katsuya

Laboratory of Sericultural Chemistry, Faculty of Agriculture, Kyushu University

https://doi.org/10.5109/23486

出版情報：九州大学大学院農学研究院紀要. 21 (1)，pp.47-66，1977-02. Kyushu University バージョン：

権利関係 : 
J. Fac. Agr., Kyushu Univ., 21, 47-66 (1977)

\title{
Computer Analysis of Lysozyme-catalyzed Reaction with Chitooligosaccharide
}

\author{
Atsushi Masaki, Yoichi Aso, Daizo Koga* \\ and Katsuya Hayashi \\ Laboratory of Sericultural Chemistry, Faculty of Agriculture, \\ Kyushu University 46-02, Fukuoka 812
}

(Received September 17, 1976)

\begin{abstract}
The scheme of the lysozyme-catalyzed reaction with chitooligosaccharides was described and the mathematical model was made on the basis of the established scheme. The model consists of simultaneous nonlinear differential equations and simultaneous nonlinear algebraic equations. The differential and algebraic equations were numerically solved by the Fowler-Warten method and the Newton method, respectively. The enzymatic reaction with tetrasaccharide (NAG), was simulated to certify the appropriateness of the reaction scheme, mathematical model and numerical computation. The minimization on the error due to the disregard of the amount of higher chitosaccharides formed by enzymatic transglycosylation was especially examined at the level of the mathematical model.
\end{abstract}

\section{INTRODUCTION}

Lysozyme (EC 3.2.1.17) is a carbohydrolase, for which the three-dimensional structure has been clearly demonstrated by X-ray analysis (Blake et al., 1967 a, b). It is well known that lysozyme exhibits a high capability of catalyzing transglycosylation. Therefore, the time-course of lysozyme-catalyzed reaction shows complicated and characteristic features, which interfere with the simple kinetic analysis of the enzymatic reaction to estimate the rate constants.

The lysozyme-catalyzed reaction with cell wall tetrasaccharides (NAG-NAM), for instance, exhibited a pronounced induction-period typical of autocatalytic reactions and a specific product-composition in the equilibrium stage. During the course of the reaction, saccharides of higher degree of polymerization than the starting substrate (NAG-NAM) $)_{2}$ appeared in the reaction mixture (Chipman et al., 1968).

Thus, the kinetic analysis of the lysozyme-catalyzed reaction requires a numerical solution of the rate equations by a computer. Chipman (1971) and Tada and Kakitani (1972) have separately formulated the mathematical models (rate equations) for the enzymatic reaction with substrate (NAG-NAM), and

* Present address : Laboratory of Biochemistry, Faculty of Agriculture, Yamaguchi University, Yamaguchi 753

Abbreviations used are : NAG, $N$-acetyl-D-glucosamine; NAM, N-acetylmuramic acid; NAG-NAM, NAG- $\beta 1 \rightarrow 4-N A M ;(N A G), \quad \beta 1 \rightarrow 4$-linked n-mer of NAG; (NAG-NAM), $\beta 1 \rightarrow$ 4-linked m-mer of NAG-NAM 
analyzed the reaction by numerical computation.

The authors have obtained some experimental results (unpublished) on the lysozyme-catalyzed reaction with substrate, chitosaccharide (NAG),.. The timecourse of the enzymatic reaction with substrate (NAG), did not show a clear induction in contrast to the case of (NAG-NAM),. Furthermore, higher chitosaccharides than the starting substrate (NAG), were also not detected. These results prompted us to estimate the rate constants in the enzymatic reaction, because the difference in rate constants observable on different substrates might produce the information for the elucidation of molecular mechanism on the high efficiency of the transglycosylation of lysozyme. It is however easily presumable that the enzymatic reaction with substrate of (NAG), type may have more complicated details than that with (NAG-NAM),: NAG-residue can bind to the six binding subsites of lysozyme; $\boldsymbol{A}, \boldsymbol{B}, \boldsymbol{C}, \boldsymbol{D}, \boldsymbol{E}$ and $\boldsymbol{F}$, while (NAG-NAM)-unit can bind only the three subsites; $\boldsymbol{A B}, \boldsymbol{C D}$ and $\boldsymbol{E F}$. Furthermore, (NAG), can be cleaved at its three different glycosidic linkages, in contrast to (NAG-NAM), which has only one cleavable linkage.

In the present study, kinetic models for the enzymatic reaction with chitooligosaccharides as substrate were made in detail according to the models reported so far by many investigators and the establishment of the computation technique for the anlysis of the model was attempted.

The present paper deals mainly with the computation technique on numerical solution of the rate equations, especially the minimization methods for the execution time and the error arisen from the disregard of higher chitosaccharides produced by transglycosylation.

\section{REACTION SCHEME}

The lysozyme-catalyzed reaction consists of three processes (Fig. 1). In the first process, the cleavage of the glycosidic linkage of substrate takes place and a C-l carbonium intermediate is formed. Then the process branches off to the two different directions ; one is the transglycosylation process and the other is the hydration process.

A typical reaction scheme for substrate (NAG), is illustrated symbolically in Fig. 2. Lysozyme has six binding subsites $\boldsymbol{A}, \boldsymbol{B}, \boldsymbol{C}, \boldsymbol{D}, \boldsymbol{E}$ and $\boldsymbol{F}$. When the enzyme binds free (NAG), $M_{4}$, original substrate, which occupies the four subsites $\boldsymbol{B}, \boldsymbol{C}, \boldsymbol{D}$ and $\boldsymbol{E}$, an enzyme-substrate complex (1: 1) $C_{4.3}$ is formd. In $\mathrm{C}_{4.3}$, the subscript 4 indicates (NAG), and 3 indicates that the 3rd NAG-residue from the nonreducing terminus of (NAG), locates on the subsite $\boldsymbol{D}$. Lysozyme cleaves the glycosidic linkage situated between the subsite $\boldsymbol{D}$ and $\boldsymbol{E}$. The (NAG), in $C_{4.3}$ form is cleaved into carbonium intermediate (NAG): and (NAG), (NAG): remains bound to the the three subsites $\boldsymbol{B}, \boldsymbol{C}$ and $\boldsymbol{D}$, and (NAG), to the subsite $\boldsymbol{E} . \boldsymbol{B}_{3.1}$ is the complex of such a (NAG): and (NAG) ${ }_{1}$ with lysozyme. The complex $B_{3,1}$ releases (NAG) ${ }_{1}$ from the subsite $\boldsymbol{E}$ and turns into a reactive intermediate $A_{3}$, which is the complex of (NAG) ${ }_{3}^{+}$with lysozyme at the same subsites. Both subsites, $\boldsymbol{E}$ and $\boldsymbol{F}$, of the intermediate are empty.

After the formation of the reactive intermediate, the process branches off 


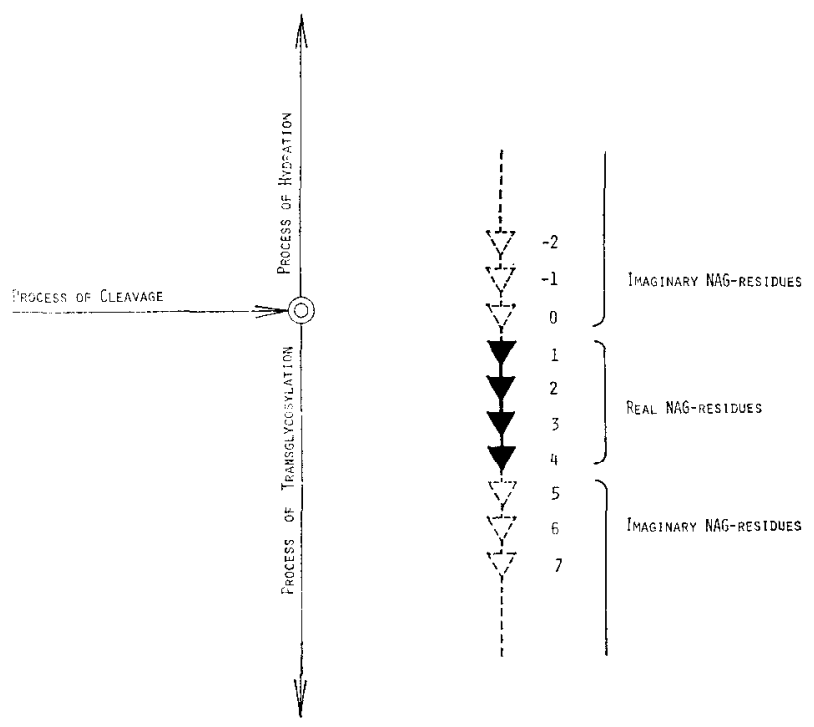

Fig. 1. Processes of the lysozyme-catalyzed reaction and the representation of substrate (NAG),

to either hydration or transglycosylation. In the hydration, the reactive intermediate A, directly reacts with water, and turns into the enzyme-substrate complex $C_{3.3} . C_{3.3}$ is the complex of (NAG), with lysozyme in which the 3rd NAG-residue from the nom-educing terminus is bound to the subsite $\boldsymbol{D}$. This complex releases (NAG), from the enzyme. Thus, the hydrolysis of (NAG), to (NAG), and (NAG) ${ }_{1}$ is conducted. When the reaction proceeds to the other type of hydration, the reactive intermediate $\mathrm{A}$, binds another free (NAG), $M_{4}$, which occupies only the subsite $\boldsymbol{F}$. A, turns into a complex $A_{3}^{4 .-1}$. The superscripe -1 means that the imaginary -1st NAG-residue of (NAM), (see Fig. 1) is located on the subsite $\boldsymbol{D}$. This intermediate complex is also reactive and reacts with water to form $C_{3.3}^{4,-1}$, which is the complex of (NAG), and (NAG), with lysozyme (1:2 complex). Then, this complex releases (NAG), and (NAG), into the medium and the hydrolysis is completed. In the transglycosylation, the reactive intermediate $A$, binds another free (NAG), $M_{4}$ as an acceptor, which occupies the subsites $\boldsymbol{E}$ and $\boldsymbol{F}$, and then turns into a complex $\boldsymbol{B}_{3.4}$, which is the complex of (NAG), with $A$,. Lysozyme can regenerate glycosidic linkage between the subsite $\boldsymbol{D}$ and $\boldsymbol{E}$, and forms new chitosaccharide (NAG) $)_{i}$. Thus, $B_{3.4}$ turns into a complex $C_{7.3}$, and (NAG), is released into the medium from the enzyme.

Another type of reaction on $(\mathrm{NAG})_{4}$, in which cleavage takes place after lysozyme has formed enzyme-substrate complex $(1: 2)$, is illustrated symbolically in Fig. 3 in order to get an easy understanding of the meaning of notations. In Figs. 2 and $3, K, K^{*}$ and $K^{\prime}$ represent binding constants, $k_{+1}, k_{-1}, k_{+2}$ and $k_{-2}$ represent rate constants of the cleavage of glycosidic linkage, the formation of glycosidic linkage, hydration and dehydration, respectively. 


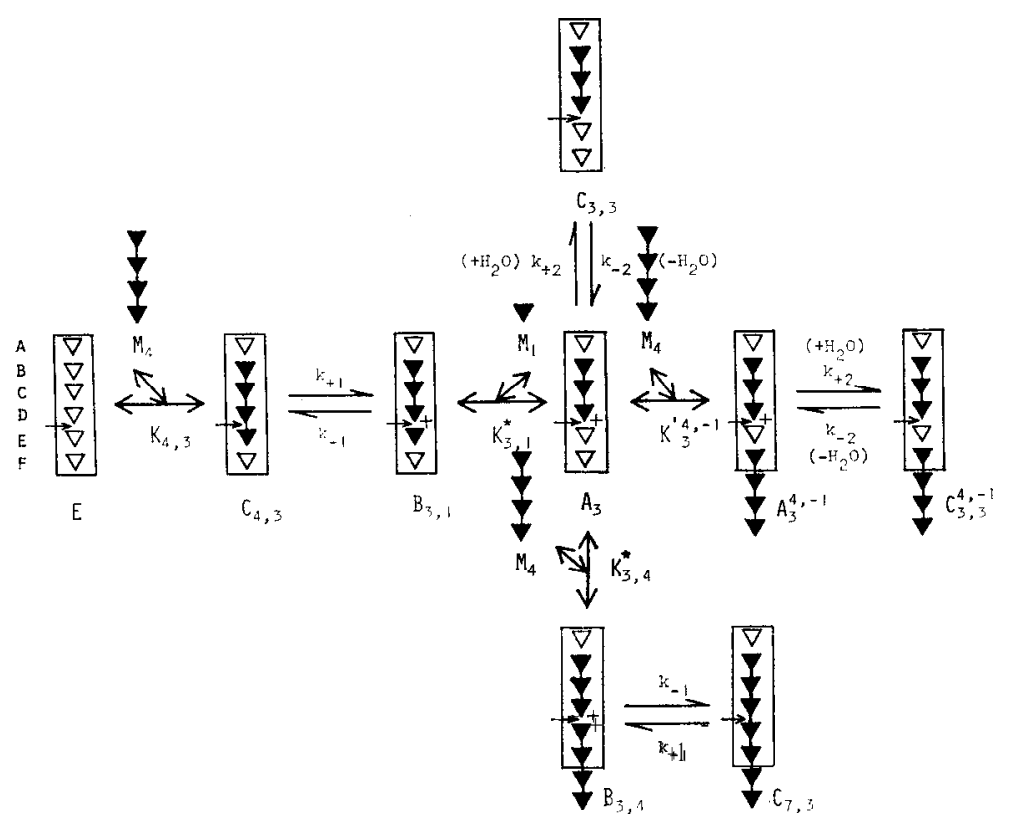

Fig. 2. Symbolic representation of a typical reaction scheme for substrate (NAG), ( $1: 1$ ES complex). The closed triangle $\boldsymbol{\nabla}$ represents a NAGresidue with its carbon 1 at the low apex. The triangle $\boldsymbol{\nabla}$ represents a carbonium intermediate of NAG-residue, in which the carbon 1 is in the form of carbonium ion. The open triangle $\nabla$ represents an empty subsite on the enzyme capable of binding a NAG-residue. The six regions are, from the top to the bottom, $\boldsymbol{A}, \boldsymbol{B}, \boldsymbol{C}, \boldsymbol{D}, \boldsymbol{E}$ and $\boldsymbol{F}$. The arrow $\rightarrow$ represents the catalytic site. The arrow $\longleftrightarrow$ represents a process in a dynamic equilibrium state. For the other notations, see text.

General reaction scheme is divided into four subschemes as shown in Figs. 4,5,6 and 7. Circles on the notations indicate typical connection points between subschemes. In construction of the general scheme, the following four assumptions were made:

(Assumption 1) The binding processes are usually in dynamic equilibrium states, because the rate constants in the binding process are known to be extremely larger than those in the catalytic processes.

(Assumption 2) The complexes from $1: 3$ to $1: 6$ are neglected because their amounts, if any, are expected to be very small throughout the reaction period. The notation $1: n$ means the molecular ratio of the enzyme to substrate in a complex. In the hydration process, however, $1: 3$ complex was partly taken into the consideration.

(Assumption 3) The catalytic rate constants are not dependent on the size of the substrate.

(Assumption 4) The reactive intermediate complexes $A_{i 1}^{j-1}$ and ${ }_{m, l_{1}} A_{i 3}^{j-1}$ cannot bind (NAG) ${ }_{1}$ to the empty subsite $\boldsymbol{E}$. 

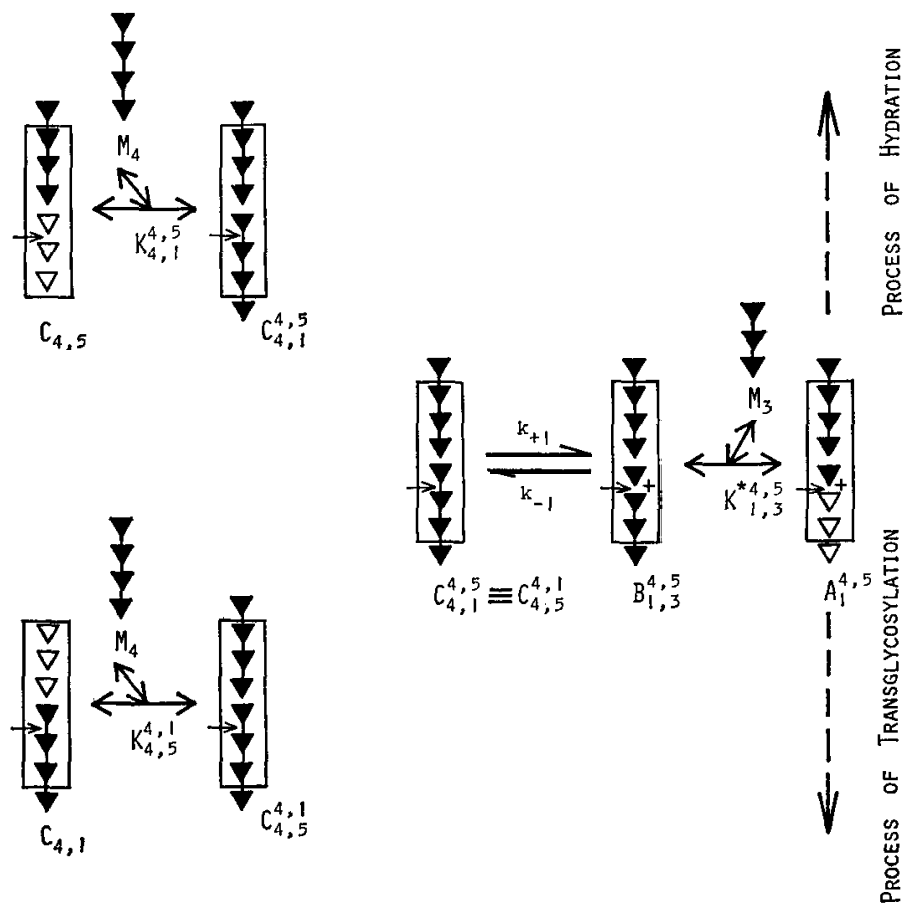

Fig. 3. Symbolic representation of a typical reaction scheme for (NAG), (1:2 ES complex). The triplet $\equiv$ means that both notations indicate the same complex.

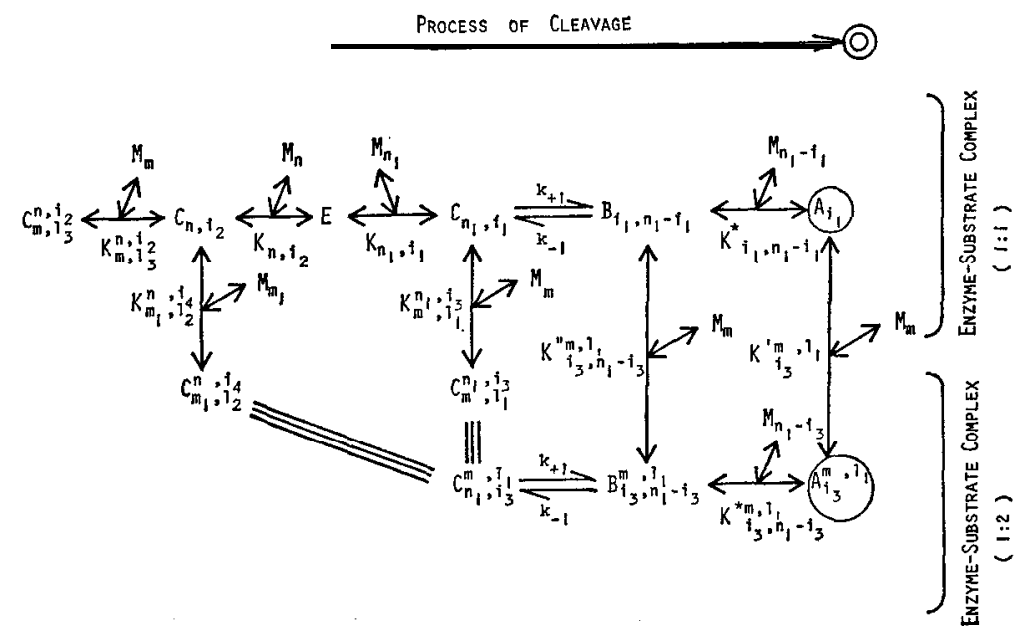

Fig. 4. Process of cleavage in the lysozyme-catalyzed reaction with chitooligosaccharide. The arrow and triplet indicate the same as those in Figs. 2 and 3 . The other notations, see text. The circles represent the connection points between the subschemes in Figs. from 4 to 7 . The entire scheme is divided into four subschemes and shown in Figs. from 4 to 7 . 


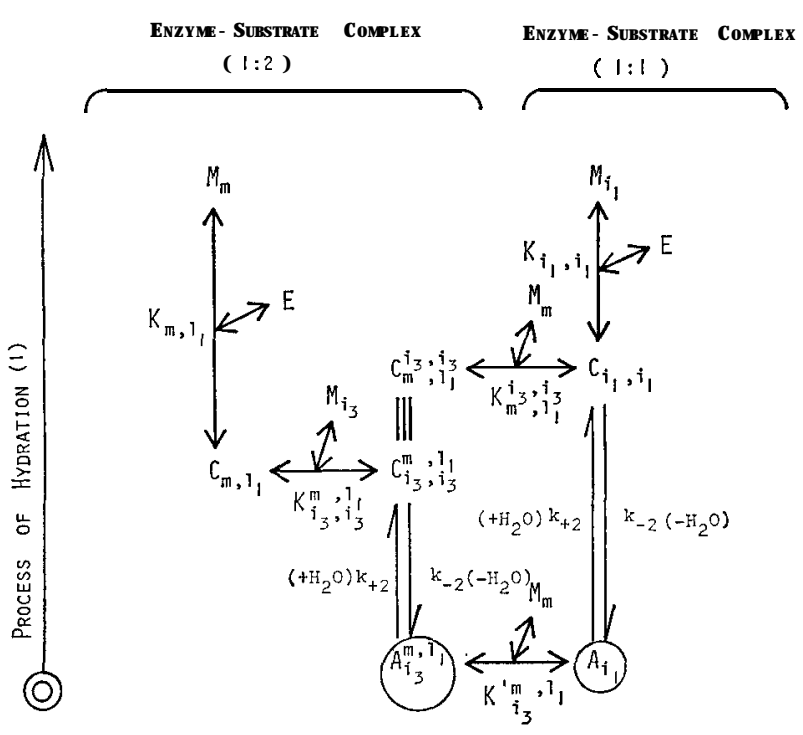

Fig. 5. Process of hydration.

The meanings of notations used in the general scheme are as follows: $C_{n, i}$ is a $1: 1$ complex of (NAG), with lysozyme. The subscript $\mathbf{i}$ indicates that i-th NAG-residue from the nonreducing terminus of (NAG), is bound to the subsite $D$. $C_{m . l}^{n . l}$ is a $1: 2$ complex of (NAG), with $C_{n \cdot l \cdot n, l} C_{s, r}^{m, t}$ is a $1: 3$ complex of (NAG)s with $C_{m, l}^{n, l}$. The suffixes $l$ and $\mathbf{r}$ have the same meaning as $\mathbf{i}$. The notation $\mathrm{C}$ represents an enzyme-substrate complex. A, is a complex of (NAG) i1 $_{1}^{+}$ with lysozyme. The $i_{1}$ th NAG-residue from the nonreducing terminus of (NAG):, is bound to the subsite $D$. $A_{i_{1}}^{m \cdot l_{1}}$ is a complex of (NAG), with $A_{i_{1}}$. ${ }_{m, l_{1}} A_{i_{1}}^{j_{1}-1}$ is a complex of (NAG); with $A_{i_{1}}^{m, l_{1}}$. The suffixes $l_{1}$ and -1 indicate the same meaning as $\mathbf{i}$ in $C_{n, i} . B_{i, j}$ is a complex of (NAG), with $A_{i}$. The 1st NAGresidue from the nonreducing terminus of (NAG), is bound to the subsite $\boldsymbol{E}$. $\boldsymbol{B}_{i, j}^{m, l}$ is a complex of (NAG),,, with $\boldsymbol{B}_{i, j}$. The notations A and B represent reactive intermediates.

There are productive and non-productive complexes in the enzyme-substrate complexes. The classification of the complexes is listed in Table 1 .

When the further two assumptions are made, the general reaction scheme can be reduced to a comparatively simple form.

(Assumption 5) The $1: 2$ and $1: 3$ complexes are neglected, because their amounts are also expected to be very small during the reaction period.

(Assumption 6) The reactive intermediate complexes $A_{i 2}^{j,-1}$ and ${ }_{m, 1_{1}} A_{i 3}^{j-1}$, which include (NAG), bound to the subsite $\boldsymbol{F}$ and have the empty subsite $\boldsymbol{E}$, a re neglected because of their small quantities.

The simple scheme, thus reduced, is shown in Fig. 8. 


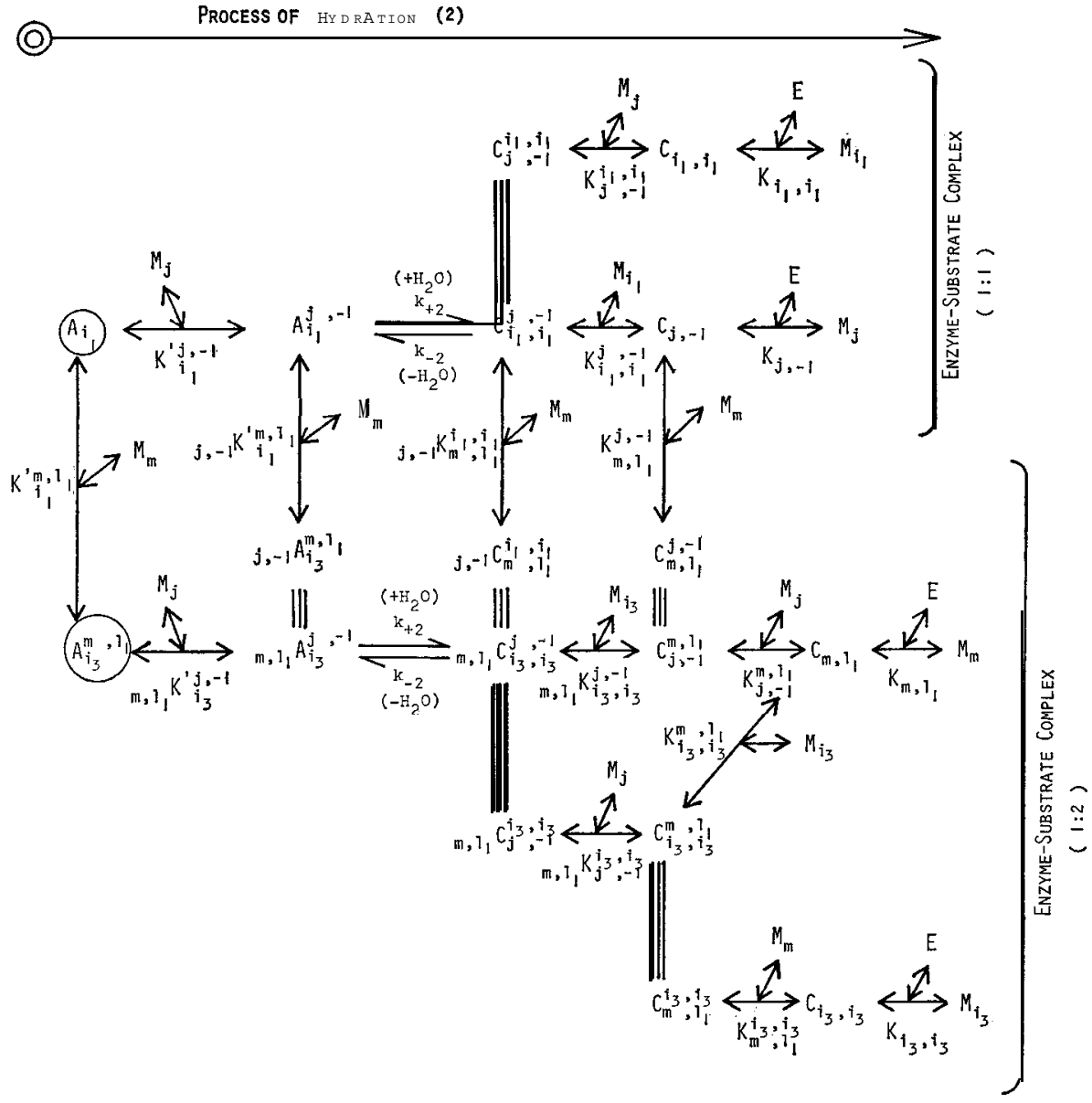

Fig. 6. Process of another type of hydration.

\section{MATHEMATICAL MODEL}

The mathematical model (rate equations) was derived from the reduced scheme shown in Fig. 8. Chitosaccharides from (NAG), to (NAG), were taken into consideration.

Let $S_{n}$ and $I_{j}$ be the total concentrations of (NAG), and (NAG); respectively. These variables may be represented as,

$$
\begin{aligned}
& S_{n}=M_{n}+\sum_{i=-1}^{n+3} C_{n, i}+\sum_{j=1}^{\mathrm{P}-1} B_{j, n} \quad(n=1,2, \cdots \cdots, p) \\
& I_{j}=A_{j}+\sum_{n=1}^{p} B_{j, n} \quad(j=1,2, \cdots \cdots, p-1)
\end{aligned}
$$




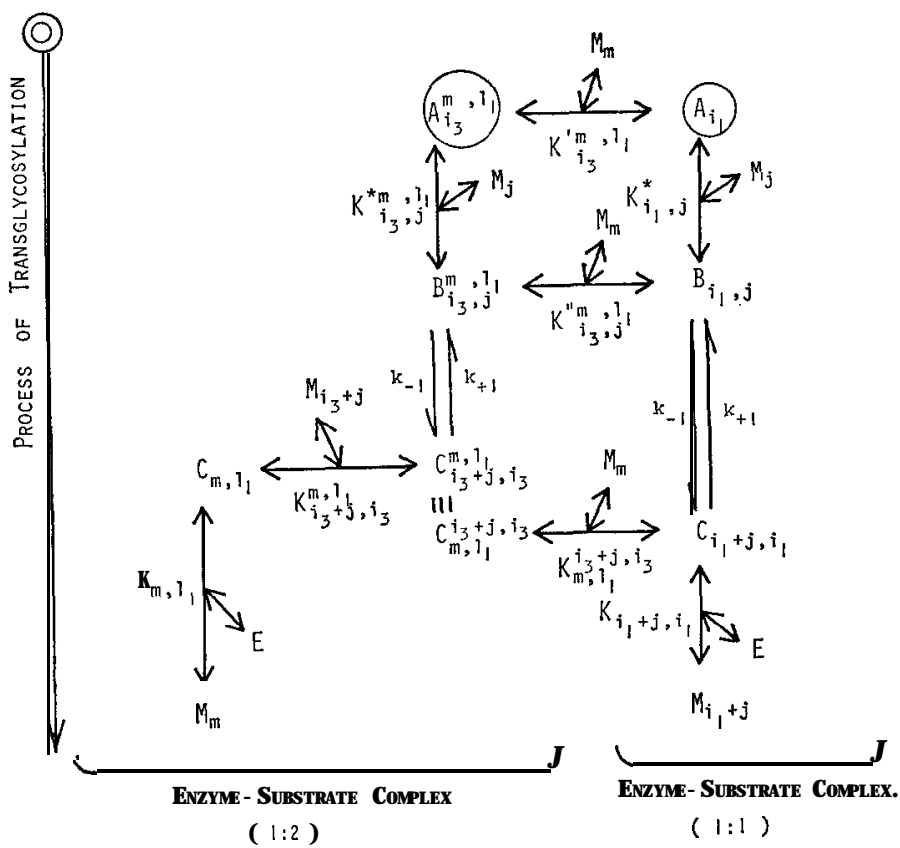

Fig. 7. Process of transglycosylation.

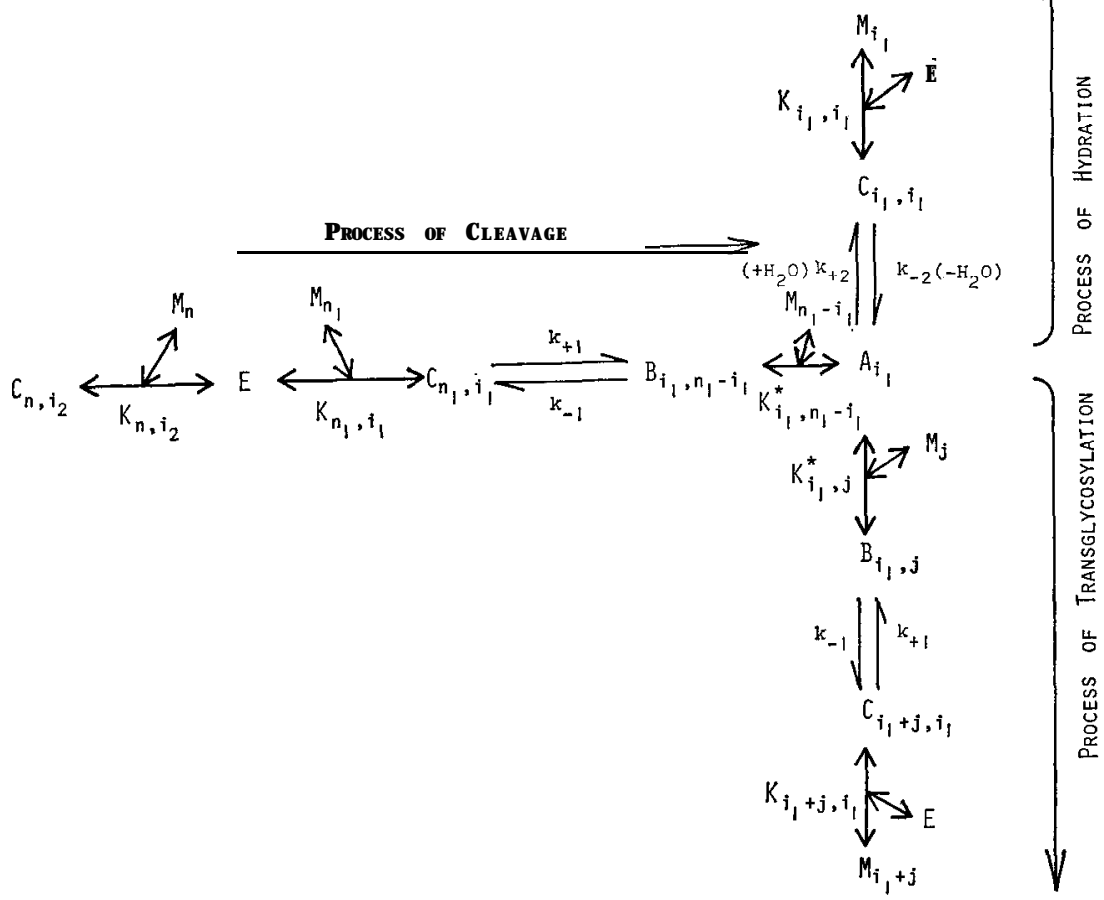

Fig. 8. The reduced reaction scheme subjected to the numerical computation. 
Table 1. Classification of the enzyme-substrate complexes.

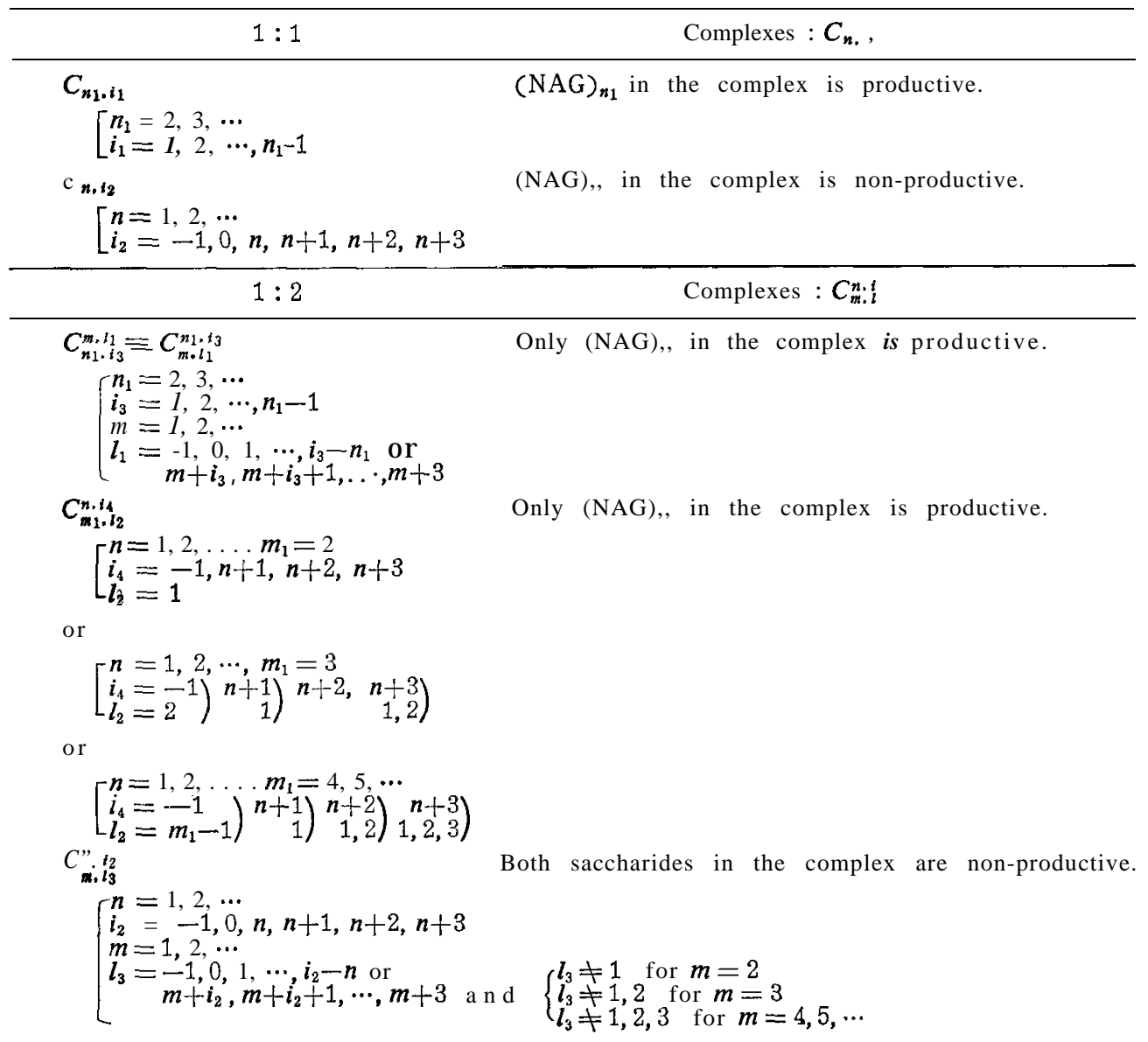

The differential equations on variables $S_{n}$ and $I_{j}$ are written as,

$$
\begin{aligned}
& \frac{d}{d t} S_{1}=k_{+2} A_{1}-k_{-1} \sum_{j=1}^{p-1} B_{j,-1}+k_{+1} \sum_{i=2}^{p} C_{i, l-1} \\
& \frac{d}{d t} S_{n}=k_{+2} A_{n}+k_{-1}\left(\sum_{m=1}^{n-1} B_{m, n-m}-\sum_{j=1}^{p-1} B_{i_{*} n}\right)+k_{+1}\left(\sum_{l=n+1}^{p} C_{l, l-n}-\sum_{i=1}^{n-1} C_{n, i}\right) \\
& (n=2,3, \cdots \cdots, p) \\
& \frac{d}{d t} I_{j}=-k_{+2} A_{j}+k_{+1} \sum_{i=j+1}^{b} C_{l, j}-k_{-1} \sum_{n=1}^{p} B_{j, n} \quad(j=1,2, \cdots \cdots, p-1)
\end{aligned}
$$
used :

In the derivation of above equations, the following assumption was also

(Assumption 7) The rate constant of dehydration is zero $\left(k_{-2}=0\right)$. There has been no experimental evidence that the dehydration process takes place in the real enzymatic reaction.

The complexes $C_{n, t}$ and $B_{j, n}$ are represented by introducing the binding 
constants as,

$$
\begin{aligned}
K_{n, i}=\frac{C_{n, i}}{E M_{n}} \quad(n=1,2, \ldots \ldots, p ; i=-1,0,1,2, \cdots \cdots, n+3) \\
K_{j, n}^{*}=\frac{B_{i, n}}{A, M_{n}} \quad(n=1,2, \cdots \cdots, p ; j=1,2, \cdots \cdots, p-1)
\end{aligned}
$$

where $K_{n, i}$ are:

In the case of $n=1$,

$$
\begin{aligned}
& K_{1,-1}=K_{F} \\
& K_{1,3}=K_{B} \\
& K_{1,0}=K_{E} \\
& K_{1,2}=K_{C}
\end{aligned}
$$

In the case of $n=2$,

$$
\begin{array}{llll}
K_{2,-1}=K_{F} & K_{2,0}=K_{E F} & K_{2,1}=K_{D E} & K_{2,2}=K_{C D} \\
K_{2,3}=K_{B C} & \mathrm{La}=K_{A B} & K_{2,5}=K_{A} &
\end{array}
$$

In the case of $n=3$,

$$
\begin{array}{llll}
K_{3,-1}=K_{F} & K_{3,0}=K_{E F} & K_{3,1}=K_{D \sim F} & K_{3,2}=K_{C \sim E} \\
K_{3,3}=K_{B \sim D} & K_{3,4}=K_{A \sim C} & K_{3,5}=K_{A B} & K_{3,6}=K_{A}
\end{array}
$$

In the case of $n=4$,

$$
\begin{array}{llll}
K_{4,-1}=K_{F} & K_{4,0}=K_{E F} & K_{4,1}=K_{D \sim F} & K_{4,2}=K_{C \sim F} \\
K_{4,3}=K_{B \sim E} & K_{4,4}=K_{A \sim D} & K_{4,5}=K_{A \sim C} & K_{4,6}=K_{A B} \\
K_{4,7}=K_{A} & & & \\
& &
\end{array}
$$

In the case of $n=5,6, \cdots \cdots, p$,

$$
\begin{array}{llll}
K_{n,-1}=K_{F} & K_{n, 0}=K_{E F} & K_{n, 1}=K_{D F} \quad K_{n, 2}=K_{C \sim F} \\
K_{n, 3}=K_{B \sim F} & K_{n, i}=K_{A \sim F} & (i=4,5, \cdots \cdots, n-2) \\
K_{n, n-1}=K_{A \sim E} & K_{n, n}=K_{A \sim D} & K_{n, n+1}=K_{A \sim C} \quad K_{n, n+2}=K_{A B} \\
K_{n, n+3}=K_{A} & &
\end{array}
$$

and $K_{j . n}^{*}$ are;

$$
K_{,, n}^{*}= \begin{cases}K_{E} & (n=1 ; j=1,2, \cdots \cdots, p-1) \\ K_{E F} & (n=2,3, \cdots \cdots, p ; j=1,2, \cdots \cdots, p-1)\end{cases}
$$

In the above formulations, the following assumption was adopted:

(Assumption 8) The binding constants are determined by the interaction between the substrate and the six binding subsites of lysozyme; $A, B, C, D, E$ and $\boldsymbol{F}$.

The constants $K_{n, i}$ and $K_{j, n}^{*}$ can be replaced by twenty-one binding constants described above. However, these binding constants may be essentially composed of less than or equal to six binding constants by means of the relationships shown in the following equation, when the additivity of binding energies (unitary standard free energy changes) is assumed (Assumption 9).

$$
\begin{aligned}
K_{X \sim Y} & =\exp \left(-\Delta F_{X \sim Y}^{0} / R T\right) \\
& =\exp \left\{-\left(\Delta F_{X \sim Y}^{u}+\Delta F_{\text {mlx }}\right) / R T\right\} \\
& =\exp \left[-\left\{\left(\Delta F_{X}^{u}+\cdots \cdot \cdot+\Delta F_{Y}^{u}\right)+\Delta F_{\text {m|x }}\right\} / R T\right]
\end{aligned}
$$

where $\Delta F_{X \sim Y}^{0}$ and $K_{X \sim Y}$ represent the standard free energy change at the site $\mathrm{X}-\mathrm{Y}$ and its binding constant. $\Delta F_{X}^{u}, \cdots, \Delta F_{Y}^{u}$ represent the binding energies at subsite $\mathrm{X}, \cdots, Y$, respectively. $\Delta F_{\mathrm{mix}}$ is the standard free energy change arisen from the mixing entropy, $R$ the gas constant, and $T$ absolute temperature.

If $\Delta F_{\text {mlx }}$ is taken to be zero (Assumption 10), $K_{X \sim Y}$ is represented by, 


$$
K_{X \sim Y}=\exp \left\{-\left(\Delta F_{X}^{u}+\cdots \cdots+\Delta_{Y}^{u} F\right) / R T\right\}
$$

The conservative equations for the total amount of substrate and enzyme may be represented as,

$$
\begin{aligned}
& \sum_{n=1}^{p} n S_{n}+\sum_{n=1}^{p-1} n I_{n}=\sum_{n=1}^{p} n M_{n}^{0} \\
& E+\sum_{n=1}^{p} \sum_{i=-1}^{n+3} C_{n, i}+\sum_{j=1}^{p-1} I_{j}=E^{0}
\end{aligned}
$$

The equations from (1) to (9) should be solved simultaneously. Some parts of the equations, however, can be transformed to more convenient forms. By introduction of equations (6) and (7), $C_{n, i}$ and $B_{j, n}$ in equations (1), (2) and (9) can be eliminated jointly. The resultant equations are,

$$
\begin{aligned}
& M_{n}+\sum_{i=-1}^{n+3} K_{n, i} E M_{n}+\sum_{j=1}^{\mathrm{P}-\mathrm{i}} K_{j, n}^{*} A_{j} M_{n}=S_{n} \quad(n=1,2, \cdots \cdots, p) \\
& A_{j}=I_{j} /\left(1+\sum_{n=1}^{p} K_{j, n}^{*} M_{n}\right) \quad(j=1,2, \cdots \cdots, p-1) \\
& E=\left(E^{0}-\sum_{j=1}^{p-1} I_{j}\right) /\left(1+\sum_{n=1}^{p} \sum_{i=-1}^{n+3} K_{n, i} M_{n}\right)
\end{aligned}
$$

Furthermore, $A$, and $E$ can be eliminated from equation (10) by using equations (11) and (12),

$$
\begin{aligned}
M_{n} & +\sum_{i=-1}^{n+3} K_{n, i}\left\{\left(E^{0}-\sum_{j=1}^{p-1} I_{j}\right) M_{n}\right\} /\left\{1+\sum_{n=1}^{p} \sum_{i=-1}^{n+3} K_{n, i} M_{n}\right\} \\
& +\sum_{j=1}^{p-1} K_{j, n}^{*} I_{j} M_{n} /\left(1+\sum_{n=1}^{p} K_{j, n}^{*} M_{n}\right)=S_{n} \quad(n=1,2, \cdots \cdots, P)
\end{aligned}
$$

Thus, finally, the nine equations $(3) \sim(8),(11) \sim(13)$ were subjected to the numerical computation.

\section{NUMERICAL COMPUTATION}

\section{General procedure}

The flow diagram of computation is shown in Figs. 9 and 10. First, the various constants, namely binding energies and rate constants, and the initial concentrations of the enzyme and substrate are read in the main program and then the binding constants are calculated from the read binding energies. Then, the flow of the calculation moves into the subprogram DIF. The simultaneous nonlinear algebraic equations (13) on $M_{n}$ are solved by the Newton method. $E$, $A, B_{f, n}$ and $C_{n . i}$ are obtained by calculations of equations (12), (11), (7) and (6), respectively. The gradients of $S_{n}$ and $I_{2}$ are finally calculated. Second, the flow of the calculation returns to the main program again and the simultaneous nonlinear differential equations on $S_{n}$ and $I_{j}$ are solved. At this step in the main program, the gradients calculated in the subprogram are used. The solved value of $S_{n}$ is written and subsequently the same calculations as the above are repeated.

The simultaneous nonlinear algebraic equations were solved by the Newton 


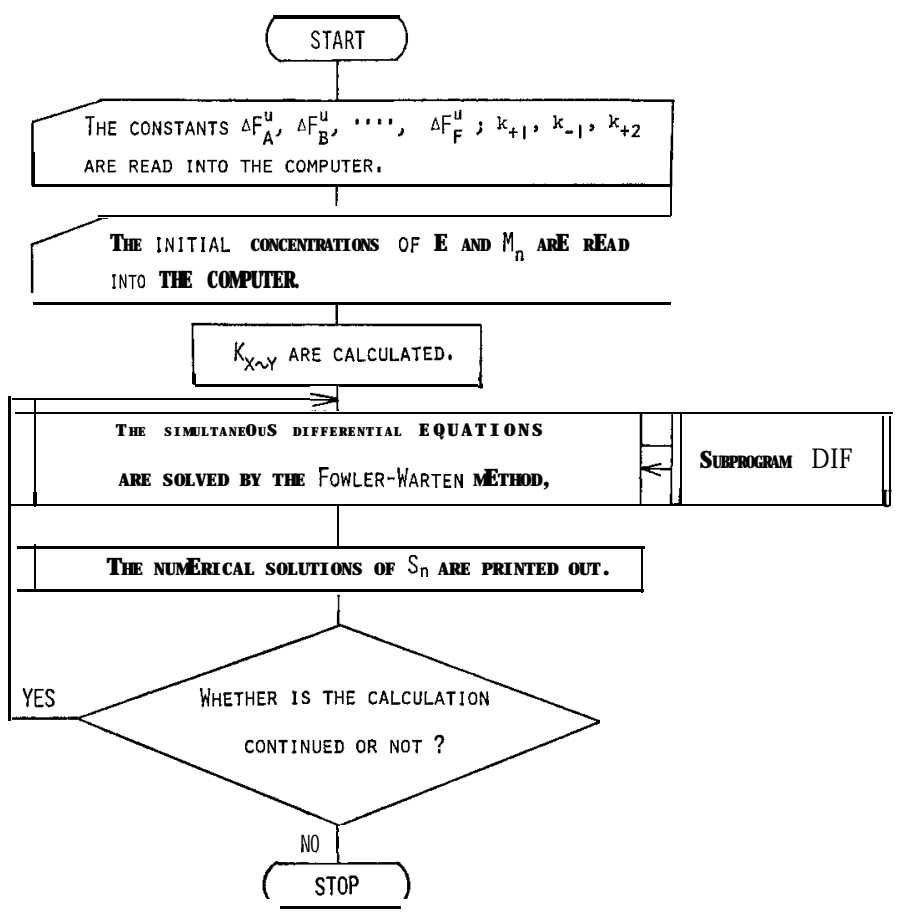

Fig. 9. Flow diagram of the main program.

SUBPROGRAM DIF

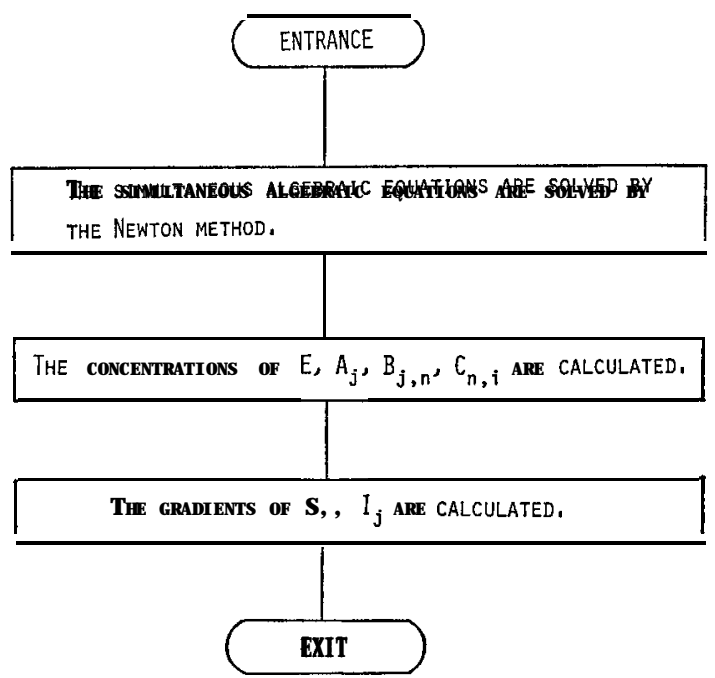

Fig. 10. Flow diagram of the subprogram DIF. 
method (FACOM scientific subroutine library D/009) and the simultaneous nonlinear differential equations were solved by the modified Fowler-Warten method (Aso et al., 1977).

\section{Parameter setting for computation}

A set of the binding energies $\Delta F_{A}^{\alpha}, \Delta F_{B}^{u}, \cdots, \Delta F_{F}^{\mu}$ and that of the rate constants $k_{+1}, k_{-1}, k_{+2}$ used in the calculation are listed in Table 2. The binding energies are the averages of many experimental values. The rate constants were predicted consulting the values of the kinetic constants obtained by Tada and Kakitani (1972). Only the rate constant $k_{-1}$ is however assumed to be $1 / 1500$ of the reported value in order to simplify the trial computation on the kinetic model. In the prediction of the rate constants, the following equation was used.

$$
k_{s}=\frac{\kappa T}{h} \exp \left(-\Delta F_{s}^{*} / R T\right) \quad(s= \pm 1,2)
$$

where $k_{s}$ and $\Delta F_{s}^{*}$ represent the rate constant and the free energy of activation. $\boldsymbol{\kappa}$ and $h$ represent Boltzman's constant and Plank's constant.

Table 2. Parameters used for computations.

\begin{tabular}{cccc}
\hline \multicolumn{2}{c}{ Binding energy" (kcal/mole) } & Rate constant $\left(\mathrm{sec}^{-1} ; 50^{\circ} \mathrm{C}\right)$ \\
\hline$\Delta F_{A}^{u}$ & -2.0 & $k_{+1}$ & 15.6 \\
$\Delta F_{B}^{u}$ & -3.0 & $k_{-1}$ & $16.7^{2)}$ \\
$\Delta F_{C}^{u}$ & -5.0 & $k+2$ & \\
$\Delta F_{D}^{u}$ & $\$ 4.5$ & & \\
$\Delta F_{E}^{u}$ & -2.5 & & \\
$\Delta F_{F}^{u}$ & -1.5 & & \\
\hline
\end{tabular}

1) Rupley et al. (1967), Dahlquist et al. (1966), Chipman et al. (1967), Pollock and Sharon (1970), Teichberg and Shinitzky (1973)

2) The value was approximately calculated to be 23,500 from the values of the kinetic constants obtained by Tada and Kakitani (1972). The value 16.7 was arbitrarily assumed in order to simplify the computation.

\section{Calculating time}

The simultaneous nonlinear differential equations were preliminarily solved by the Runge-Kutta-Gill method. This method, however, required a very long computation time, because it requires four repeated calculations for the gradient at each step. Accordingly, the modified Fowler-Warten method, which calculates the gradient only two times, was adopted, The calculating time was saved to about half of that required by the Runge-Kutta-Gill method.

On the other hand, the simultaneous nonlinear algebraic equations were solved by the Newton method as follows : The subroutine program was modified so that the number of iteration was reduced to at least two according to the judgement whether the difference in the calculated values between the consecutive two steps is within the value for the convergence test, $5 \times 10^{-2}$, or not. The reduction of the number of iteration and the presumption of the loose value for the convergence test were allowed by the fact that in the present 
computation very reasonable values could be used as the initially assumed values for the numerical solutions. Thus, the modification of the Newton method shortened again the calculating time.

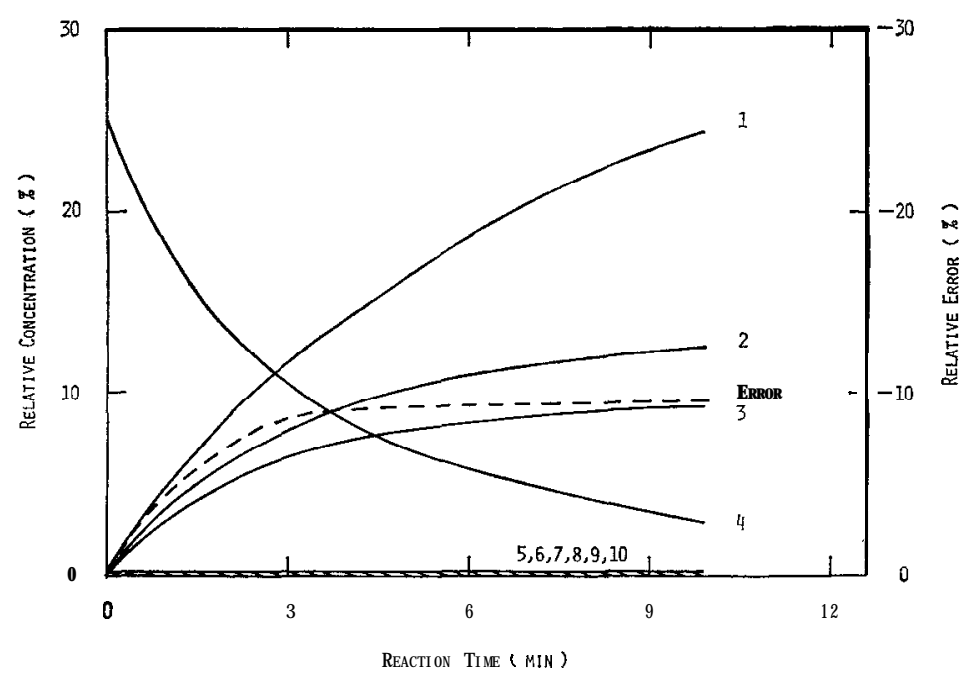

Fig. 11-a. The computed time-courses of the lysozyme-catalyzed reaction with (NAG), The values of parameters used arc listed in Table 3 . The value of $n(\mathrm{n}=1,2, \cdots, 10)$ represents (NAG), and Error represents the relative error on the sum of NAG-residue. The curves for saccharides from $(\mathrm{NAG})_{5}$ to $(\mathrm{NAG})_{10}$ lie within the shadowed portion. The magnified profiles are shown in Fig. 11-b.

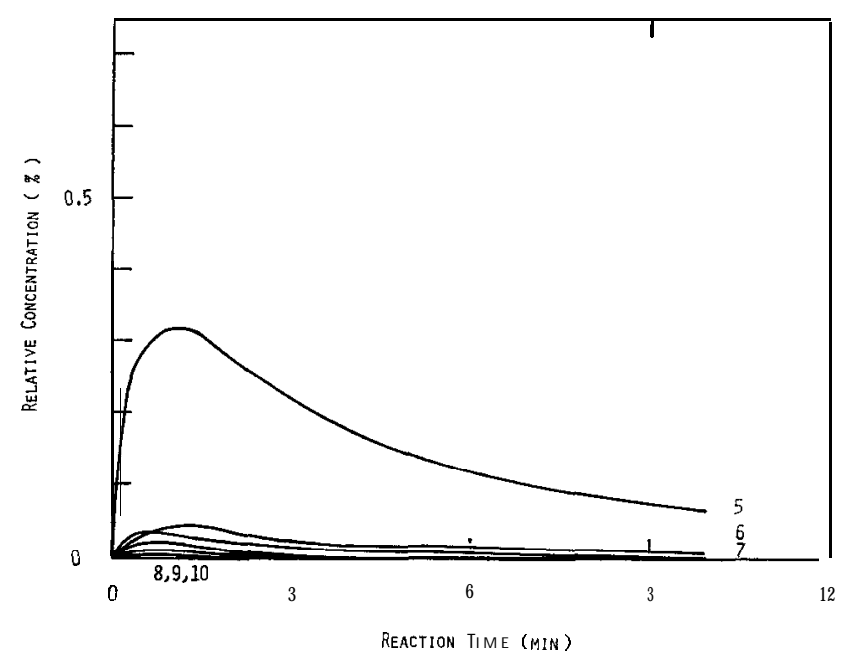

Fig. 11-b. The computed time-courses of saccharides from $(\mathrm{NAG})_{5}$ to $(\mathrm{NAG})_{10}$. 
Figure 11 shows an example of calculated results. The conditions for computation are listed in Table 3. Error in the figure indicates the relative error $(\%)$ on the sum of NAG-residue defined by the following equation (also see Eq. (8)).

$$
\text { Error }=\left[\left\{\left(\sum_{n=1}^{p} n S_{n}+\sum_{n=1}^{p-1} n I_{n}\right)-\sum_{n=1}^{p} n M_{n}^{0}\right\} / \sum_{n=1}^{D} n M_{n}^{0}\right] \times 100
$$

The error became fairly large (about $-10 \%$ ) after $12 \mathrm{~min}$. In this case, however, only the chitosaccharides from $(\mathrm{NAG})_{1}$ to $(\mathrm{NAG})_{10}$ were taken into the calculation ; $p$ was taken to be equal to ten.

Table 3. Conditions for computation.

\begin{tabular}{lc}
\hline Lysozyme & $8.15 \times 10^{-5}[\mathrm{M}]$ \\
$(\mathrm{NAG})_{4}$ & $1.05 \times 10-3$ [M] \\
Rate constants & Listed in Table 2 \\
Binding energies & Listed in Table 2 \\
$P$ & 10 \\
Factor for $E$ introduced in the modified Fowler-Warten method & 1000
\end{tabular}

\section{Minimization of relative error}

In the above calculation, chitosaccharides from (NAG) 1 to (NAG) 10 were taken into account. However, chitosaccharides from (NAG), to (NAG) $)_{19}$ synthesized by transglycosylation were neglected. Since the amounts of such higher chitosaccharides were excluded from the calculation system, it is easily presumed that this will cause a considerably large error.

The pathway of transglycosylation in the reaction scheme (Fig. 8) was partly modified in order to minimize the calculation error as follows:

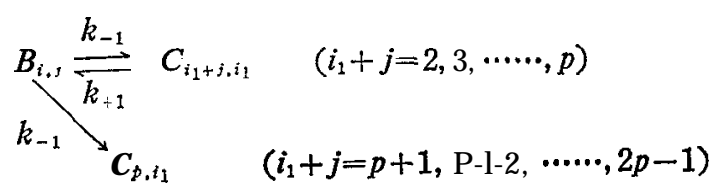

When $\mathbf{p}$ is taken to be equal to 10 , chitosaccharides from (NAG) ${ }_{11}$ to (NAG) ${ }_{19}$ are picked up as $(\mathrm{NAG})_{10}$ in this revised reaction scheme. The mathematical model is also revised and equation (4) was replaced by equations (4') and (4") ;

$$
\begin{gathered}
\frac{d}{d t} S_{n}=k_{+2} A_{n}+k_{-1}\left(\sum_{m=1}^{n-1} B_{m, n-m}-\sum_{j=1}^{p-1} B_{j, n}\right) \\
+k_{+1}\left(\sum_{l=n+1}^{p} C_{l, l-n}-\sum_{i=1}^{n-1} C_{n, i}\right) \\
(\mathrm{n}=2,3, \cdots \cdots, \mathrm{p}-\mathrm{l}) \\
\frac{d}{d t} S_{p}=\left(\frac{d}{d t} S_{n}\right)_{n=p}+\quad k_{n-p}^{2 p-1} \sum_{n+1} \sum_{m=n-p}^{p-1} B_{m, n-m}
\end{gathered}
$$

Figure 12 shows the computed result. The conditions for computation are the same as those in Fig. 11. The error decreased to about $-2 \%$. 


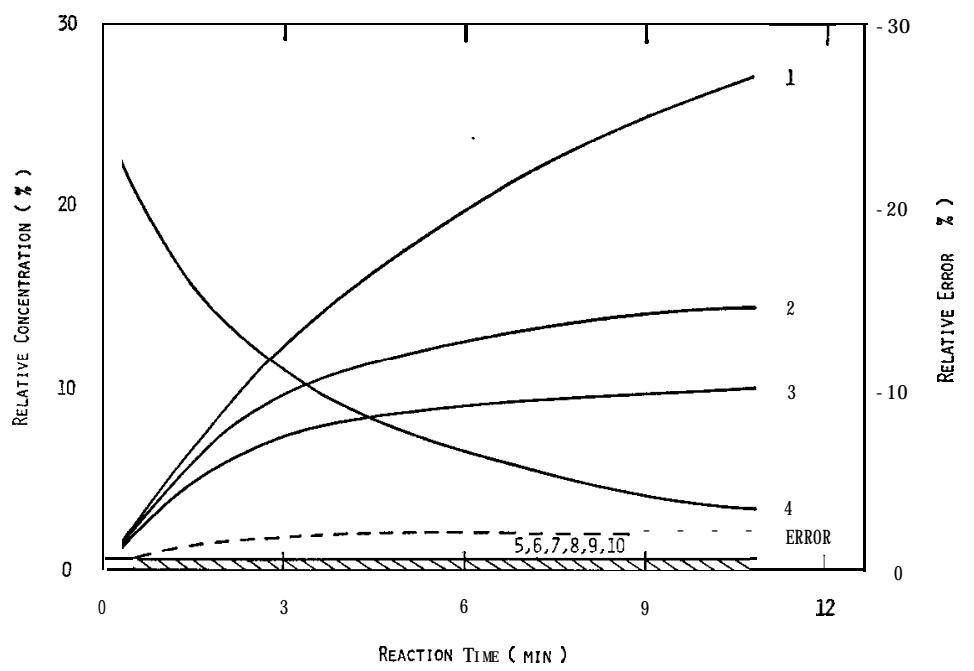

Fig. 12-a. The computed time-courses of the lysozyme-catalyzed reaction with (NAG),. Mathematical model for the process of transglycosylation was revised (see text). The values of parameters used are listed in Table 3. The value of $n(n=1,2, \cdots \cdots, 10)$ represents $(\mathrm{NAG})$,. The curves for saccharides from (NAG), to (NAG) 10 are shown in Fig. 12-b.

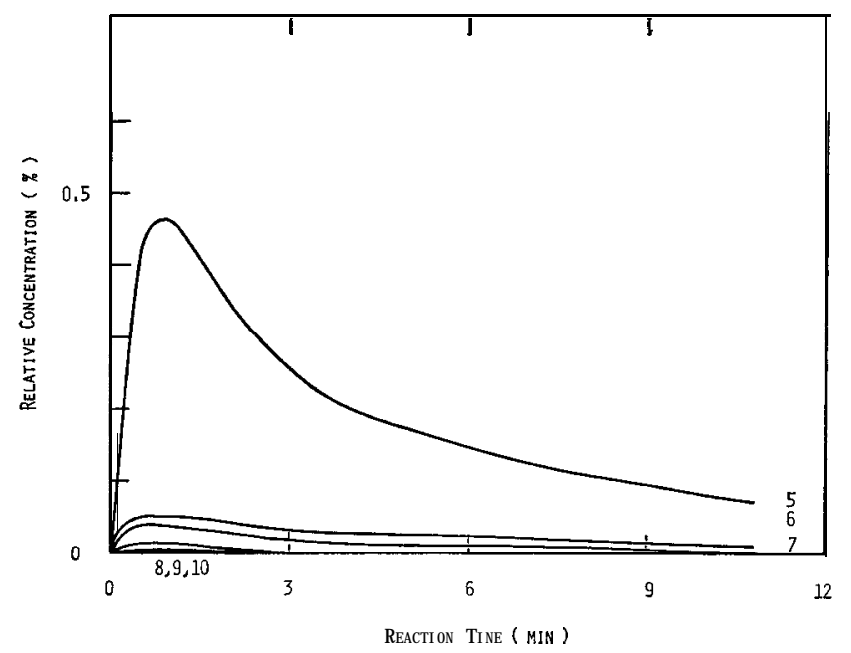

Fig. 12-b. The computed time-courses of saccharides from (NAG), to $(\mathrm{NAG})_{10}$. 


\section{DISCUSSION}

The time-course of the enzymatic reaction on substrate (NAG), exhibits a very complicated profile, as compared with that on (NAG-NAM),. Concomitantly, the reaction scheme and the mathematical model have also complicated forms. These facts suggest that the numerical analysis of the mathematical model requires a long computation time. In the present study, it was verified that the modified Fowler.Warten method was much preferable for solving the simultaneous nonlinear differential equations, instead of the Runge-Kutta-Gill method. Furthermore, the conditions for the convergence in the Newton method, which was used to solve the simultaneous nonlinear algebraic equations, were examined by means of solving practically the algebraic equations, derived in the present study, and the weakening of convergence conditions was attained. Thus, a standard computation technique, which is practically applicable with respect to the computation time for solving rate equations, could be established.

When higher chitosaccharides produced by transglycosylation were neglected and excluded from the calculating system, the error on the conservation of the substrate readily ran up to about $-10 \%$. This is because there is essentially no limit in the size of the chitosaccharides synthesized by transglycosylation. If higher chitosaccharides were taken into account as far as possible, the error will become negligible. However, it is practically difficult to include all chitosaccharides in the calculation, because the necessary computation process exceeds the capacity of computer. Then, the process of transglycosylation was modified so that the practical minimization on the error could be attained. As a result, computation error was considerably lowered to about $-2 \%$.

In the computations described above, the small value of $k_{-1}$ was assumed. However, the real value of $k_{-1}$ was thought to be very large (about $10^{3}$ times larger than $k_{-2}$ ) (Tada and Kakitani, 1972).

When such a large value is used, the simultaneous nonlinear differential equations to be solved become so-called stiff differential equations. Figure 13 shows the computed result with a large value of $k_{-1}$. The calculating conditions are listed in Table 4. The error increased to about $-25 \%$ at the late stage of the reaction. The large value of the rate constant of transglycosylation $k_{-1}$ consequently causes the production of large amount of the chitosaccharides from (NAG), to (NAG),,. Even the approximation technique to pick up higher chitosaccharides $\left((\mathrm{NAG})_{11} \sim(\mathrm{NAG})_{19}\right)$ as $(\mathrm{NAG})_{10}$ seems to cause such a large error. If the picking up approximation is improved to include much higher chitosaccharides in the mathematical model: for instance, the differential equations are formulated up to $(\mathrm{NAG})_{15}$ and chitosaccharides from (NAG) ${ }_{16}$ to $(\mathrm{NAG})_{29}$ are picked up as (NAG) ${ }_{15}$ (this means that the value of $p$ in equations (1)-(13) is taken to be 15), the error will be certainly lowered. Such an improvement on approximation technique will be easily attained.

Although lysozyme has been characterized by its great capability of transglycosylation, the molecular mechanism by which the great capability is derived is not yet fully understood. Chipman (1971) and Tada and Kakitani (1972) first estimated separately the rate constants of hydration and transgly- 


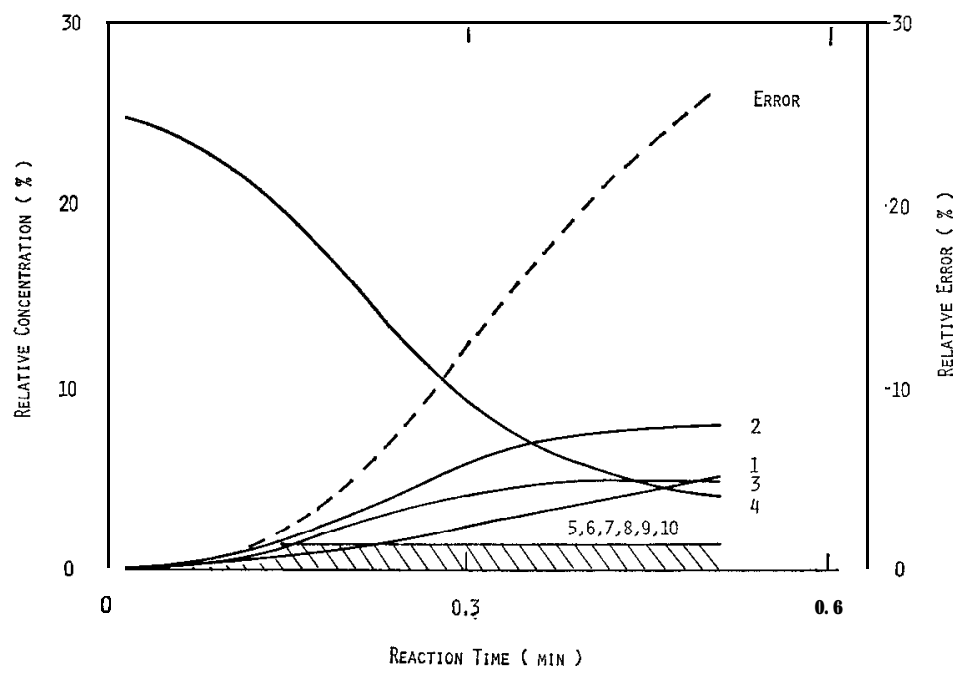

Fig. 13-a. The computed time-courses of the lysozyme-catalyzed reaction with (NAG) $)_{4}$. The value of $k_{-1}$ used is $6.00 \times 10^{6}$ and the values of the other rate constants are the same as those in Fig. 12 (listed in Table 4). The value of $n(n=1,2, \cdots \cdots, 10)$ represents $(\mathrm{NAG})$.

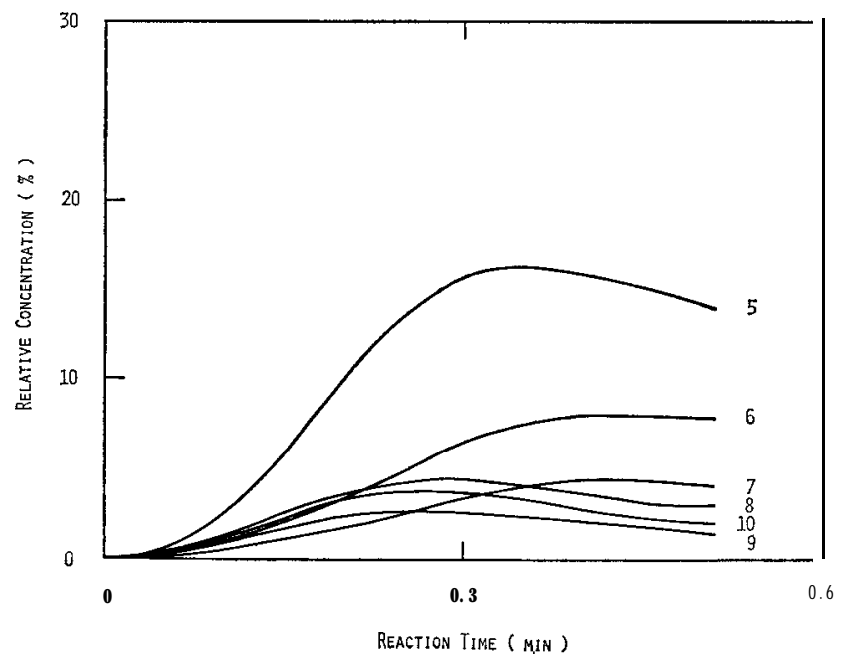

Fig. 13-b. The computed time-courses of saccharides from (NAG) $)_{5}$ to $(\mathrm{NAG})_{10}$.

cosylation using (NAG-NAM), as substrate. The enzymatic reaction with (NAG), as substrate seems to give more detailed information for elucidating the molecular mechanism on transglycosylation. Since the enzymatic reaction with (NAG), has more complicated reaction scheme than that with (NAG$\mathrm{NAM})_{n / 2}$, the separate estimation of the rate constants from experimental data 
Table 4. Conditions for computation. The process of transglycosylation in the reduced reaction scheme was modified.

\begin{tabular}{lc}
\hline Lysozyme & $8.15 \times 10^{-5}[M]$ \\
(NAG) & $1.05 \times 10-3[M]$ \\
$k_{+1}$ & $15.6 \quad\left[\mathrm{sec}^{-1} ; 50^{\circ} \mathrm{C}\right]$ \\
$k_{-1}$ & $1670 . \quad\left[\mathrm{sec}^{-1} ; 50^{\circ} \mathrm{C}\right]$ \\
$k_{+2}$ & $5.23\left[\mathrm{sec}^{-1} ; 50^{\circ} \mathrm{C}\right]$ \\
Binding energies & Listed in Table 2 \\
P & 10 \\
Factor for $E$ introduced in the modified Fowler-Warten method 1000 \\
\hline
\end{tabular}

has not yet attempted. Now, the authors are working on the separate estimation of the rate constants in the enzymatic reaction with (NAG)., using the computation technique reported in the present paper and an appropriate optimization technique.

\section{ACKNOWLEDGEMENTS}

This research was supported by a grant of scientific research from the Ministry of Education.

\section{REFERENCES}

Aso, Y., Y. Tsuji and K. Hayashi 1977 Application of the Fowler-Warten method to a stiff enzymatic reaction. J. Fac. Agr., Kyushu Univ., 21: 33-45

Blake, C. C. F., L. N. Johnson, G. A. Mair, A. C. North, D. C. Phillips and V. R. Sarma 1967a Crystallographic studies of the activity of hen egg-white lysozyme. Proc. Roy. Soc. (London), B 167: 378-388

Blake, C. C. F., G. A. Mair, A. C. T. North, D. C. Phillips and V. R. Sarma 1967b On the conformation of the hen egg-white lysozyme molecule. Proc. Roy. Soc. (London), B167: 365-377

Chipman, D.M. 1971 A kinetic analysis of the reaction of lysozyme with oligosaccharides from bacterial cell walls. Biochemistry, 10: 1714-1722

Chipman,D. M., V. Grisaro and N. Sharon 1967 The binding of oligosaccharides containing $N$-acetylglucosamine and $N$-acetylmuramic acid to lysozyme. J. Biol. Chem., 242: 43884394

Chipman, D. M., J. J. Pollock and N. Sharon 1968 Lysozyme-catalyzed hydrolysis and transglycosylation reaction of bacterial cell wall oligosaccharides. J. Biol.Chem., 243: 487-496

Dahlquist, F. W., L. Lao and M. A. Raftery 1966 On the binding of chitin oligosaccharides to lysozyme. Proc. Natl. Acad. Sci. U. S., 56: 26-30

Pollock, J. J. and N. Sharon 1970 Studies on the acceptor specifity of the lysozyme-catalyzed transglycosylation reaction. Biochemistry, 9: 3913-3925

Rupley, J. A., L. Butler, M. Gerring, F. J. Hartdegen and R. Pecorano 1967 Studies on the enzymic activity of lysozyme, III. The binding of saccharides. Proc. Natl. Acad. Sci. U. S., 57: 1088-1095

Tada, H. and T. Kakitani 1972 Theoretical aspects on structure and interaction -Lysozyme and rhodopsin as examples-. Seibutsu Butsuri (in Japanese), 12: 97-113 
A. M asaki et al.

Teichberg, V. I. and M. Shinitzky 1973 Fluorescence polarization studies of lysozyme and lysozyme-saccharide complexes. J. Mol. Biol., 74 : 519-531 